\title{
Muscle-based perception: theory, research and implications for rehabilitation
}

\author{
Percepção baseada nos músculos: teoria, pesquisa e implicações na reabilitação
}

Carello C, Silva PL, Kinsella-Shaw JM, Turvey MT

\begin{abstract}
Background: Muscle-based perception of the spatial properties of limbs constrains the patterning, timing and magnitude of muscle forces while performing motor activities. The centrality of muscle-based perception to both ordinary and skilled actions warrants attention from the rehabilitation community, since deficits in its functioning would be related to important functional limitations. In this overview, we summarize a body of research that may be used to guide the development of effective assessment tools and rehabilitation programs that are specifically directed towards such deficits. Objectives: There were four specific aims: first, to present an informationbased approach to muscle-based perception that is grounded in physical laws; second to identify central principles underlying musclebased perception that have been revealed and supported by empirical work; third, to summarize reports that have investigated whether the principles identified can be generalized to muscle-based perception in individuals with sensory-motor impairments; and fourth to provide a preliminary discussion of the potential implications of the research presented here for issues relating to rehabilitation.
\end{abstract}

Key words: perception; ecological psychology; stroke, neuropathy.

\section{Resumo}

Introdução: A percepção muscular das propriedades espaciais dos membros restringe o padrão, período e magnitude das forças exercidas durante a execução de atividades motoras. A importância central da percepção muscular, tanto para ações rotineiras quanto para ações especializadas, merece atenção da comunidade envolvida na área de reabilitação, uma vez que alterações em suas funções podem estar relacionadas a importantes limitações funcionais. Nesta revisão, os autores apresentam um resumo da pesquisa que pode ser utilizada para guiar o desenvolvimento de ferramentas de avaliação eficazes bem como programas de reabilitação que sejam especificamente direcionados para estas disfunções. Objetivos: Quatro pontos específicos foram incluídos: primeiro, a apresentação da abordagem com base em informações relativas à percepção muscular de acordo com as leis da física; segundo, a identificação dos princípios centrais determinantes da percepção muscular que vem sendo revelada e apoiada por trabalhos empíricos; terceiro, um resumo dos relatos que investigaram e se os princípios identificados poderiam ser generalizados para a percepção muscular dos indivíduos com alterações motoras e sensitivas; e quarto, uma discussão preliminar sobre as implicações potenciais da pesquisa aqui apresentada, no tocante aos assuntos relacionados à reabilitação.

Palavras-chave: percepção; psicologia ecológica; acidente vascular cerebral; neuropatia.

Received: 08/07/2008 - Revised: 09/07/2008 - Accpeted: 11/07/2008

Center for the Ecological Study of Perception and Action, University of Connecticut, Storrs (CT), United States

Correspondence to: Claudia Carello, Department of Psychology, University of Connecticut, 406, Babbidge Road, Unit 1020, 06269-1020, Storrs (CT), United States,

e-mail: claudia.carello@uconn.edu 


\section{Introduction $\because: 8$}

Haptic perception is defined as the awareness of the body and the environment by means of the body, that is, by means of mechanical contact ${ }^{1}$. Such mechanical contact is made possible by at least three different forms of touch: cutaneous, haptic and dynamic touch ${ }^{1}$. Typically, references to touch are references to the first two types: experiences obtained when objects contact the skin (cutaneous touch) or when the hands are moved over surfaces (haptic touch). The third type, dynamic touch, refers to the awareness of magnitudes and directions of implements and limbs by means of muscular effort. Arguably, this last category is the most common form of touch, albeit the least apparent ${ }^{2}$.

Dynamic touch (variously referred to as effortful touch, kinesthetic touch or muscle-based perception) is operating whenever one takes hold of something and moves it in some fashion, for example, when one lifts a cup, carries a book, or wields a baton. It is also active when tools or implements are used to act upon or explore the environment. Additionally, it functions in a more continuous way in the assembling, transforming and maintaining of posture ${ }^{3}$. Hence, muscle-based perception underlies our ability to achieve and maintain orientation of the body, limbs, and implements while visually attending to something else. This ability is relevant to the performance of countless everyday tasks, including tasks embedded within other tasks (e.g., carrying a cup of coffee while walking through cluttered surroundings, swinging a hammer while visually focused on the nail, and maintaining posture while talking to a friend). Such ubiquity suggests that deficits in muscle-based perception may well result in important functional limitations.

Despite the immersion of muscle-based perception in activity, clinical evaluations of deficits in touch typically do not reflect a functional context. Evaluations are generally restricted to more passive abilities such as perceiving and replicating joint angles in the absence of vision. Given the broad range of functional tasks involving muscle-based perception, such an assessment tool can, at best, only partially inform about the nature of that system's (diminished) involvement. Efforts to evaluate functional capacities have, perhaps incidentally, led to the development of tasks with a dynamic touch component such as a pick-up test ${ }^{4-6}$ or variants that require, in addition, placing or tossing the object ${ }^{7,8}$. These tasks emphasize manual dexterity, however, and do not address larger scale transporting and maneuvering of objects (cups, books, briefcases) and implements (spatulas, hammers, brooms). Interestingly, some clinical tests do, in fact, require transporting and maneuvering objects in functional contexts (e.g., Action research arm test ${ }^{9}$ and Wolf motor function test ${ }^{10}$. Although intact dynamic touch could facilitate or even ensure success, these tests are designed to assess functional deficits arising from pathological changes in tone and recruitment. Guided by that goal, therefore, the tests provide indices only of upper extremity motor fluency (e.g., movement execution time, error rate). For the most part, the kinds of changes in muscle-based perception that accompany sensory and motor impairments are largely unknown and far from obvious.

It might be argued that the centrality of muscle-based perception to actions both mundane and skilled warrants more attention from the rehabilitation community. Pointedly, it is not even known whether or not sensory and motor impairments result in deficits in dynamic touch. The development of effective assessment tools - let alone rehabilitation programs specifically directed at such deficits - requires a sound theory of the functioning of dynamic touch. In this overview, we summarize a body of research that provides the groundwork for needed theorizing on the muscle-based capabilities of the haptic perceptual system. There are four specific aims: first, to present an information-based approach to muscle-based perception that is grounded in physical law; second, to identify central principles underlying muscle-based perception that have been revealed and supported by empirical work; third, to summarize reports investigating whether the identified principles generalize to muscle-based perception in individuals with sensory-motor impairments; and fourth, to provide a preliminary discussion of the potential implications of the presented research for issues in rehabilitation.

\section{Informational basis for dynamic touch $: \because$.}

It has been suggested that dynamic touch exploits the physics of rotation and extracts mechanical invariants specific to hand-held objects in order to perceive their relevant properties $^{11}$. More specifically, whenever a person wields a grasped object, the hand movements, together with the physical properties of the object, produce torques, angular motions, and muscle deformations that change over time. Importantly, there are invariant parameters that relate these variable torques and motions, namely, the moments of the object's mass distribution. Such parameters are specific to the object's inertial properties and, hence, constitute viable informational variables underlying perception of its unchanging dimensions.

One can develop intuitions about an object's mass moments by imagining a hammer cut into innumerable small pieces that are left arranged so as to preserve its original shape (Figure 1). Mass, the zeroth moment, is calculated by simply adding up the masses of all those pieces. The calculation of first and second moments (static moment and moment of inertia, respectively) 


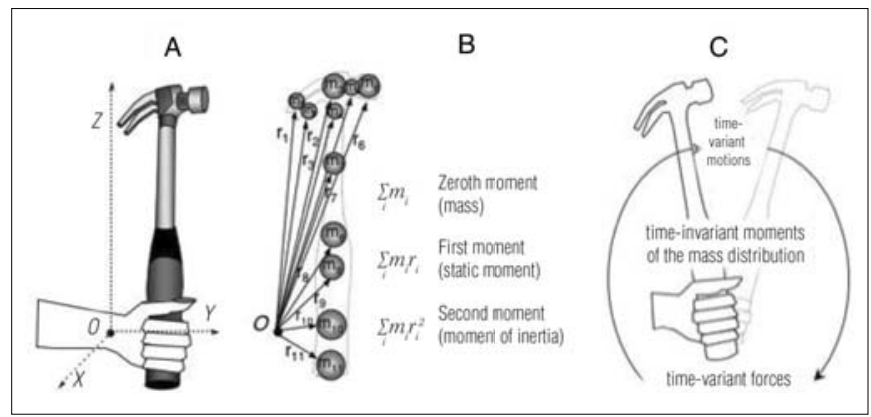

Figure 1. (A) An object grasped firmly in one hand is referred to an $x y z$ coordinate system with an origin 0 (the point of rotation in the wrist); (B) the object can be characterized as an number of masses $m_{i}$ each at a distance $r_{i}$ from 0 , thereby allowing calculation of the moments of the mass distribution; $(\mathrm{C})$ moments of the mass distribution are invariants linking motions and torques.

requires choosing an axis, say, through the end of the handle. The first moment is calculated by measuring how far a given piece is from the axis, multiplying that distance by the mass of the piece, and summing all those mass $\mathrm{x}$ distance products. Calculation of the second moment is similar, except that distance is squared before multiplication. For a hammer that is grasped by its handle, the force required to hold it vertically is proportional to mass; the force required to hold it horizontally is proportional to first moment. The second moment or moment of inertia relates to the forces required to rotate the object in different directions. Hence, a single number cannot capture its general form. Its expression requires a tensor, commonly referred to as the inertia tensor.

The inertia tensor $\left(\mathrm{I}_{\mathrm{ij}}\right)$ quantifies an object's resistance to rotation in different directions. It is represented mathematically by a symmetric $3 \times 3$ matrix: The diagonal terms (moments of inertia) capture the object's rotational inertia (resistance to rotational acceleration) with respect to three orthogonal axes centered at a point of reference $\mathrm{O}$; the off-diagonal terms (products of inertia) capture the object's rotational inertia in directions perpendicular to the axial rotations (Figure 2A). The products of inertia reflect asymmetries of the object's mass distribution about axes defined at O. A particular set of orthogonal axes can be chosen such that the object's resistance to rotational acceleration about $\mathrm{O}$ is evenly distributed (Figure $2 \mathrm{~B}$ ); with these so-called symmetry axes, the products of inertia disappear, thereby rendering the inertia tensor in its diagonal form (Figure 2C) ${ }^{12}$. The diagonalized tensor comprises the symmetry axes or eigenvectors $\mathrm{e}_{1}, \mathrm{e}_{2}$, and $\mathrm{e}_{3}$, and the principal moments of inertia or eigenvalues $\mathrm{I}_{1}, \mathrm{I}_{2}$, and $\mathrm{I}_{3}$, which quantify resistances to rotational acceleration about those axes (where the subscripts one, two, and three identify largest, intermediate and smallest resistances, respectively). Hence, the eigenvectors constitute a non-arbitrary coordinate system defined by the object itself - specifically by how the object's mass is distributed relative to the rotation point ${ }^{2}$. The implication is that both the eigenvalues and eigenvectors are relevant mechanical

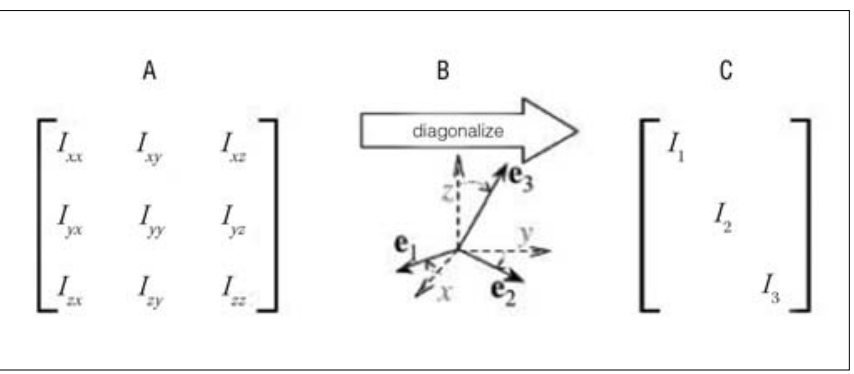

Figure 2. (A) An inertia tensor defined as a $3 \times 3$ matrix. On the diagonal are the resistances to rotational acceleration about the $x, y$, and $z$-axes. Off the diagonal are resistances perpendicular to these (influenced by how asymmetrically the mass is distributed; (B) any xyz-coordinate system can be referred to the symmetry axes $e_{k}$, termed the eigenvectors; (C) this transform renders the off-diagonal components equal to zero and leaves the principal moments of inertia or eigenvalues.

invariants specific to and, hence, potentially informative about an object's persistent properties.

The typical paradigm investigating the inertial dependency of muscle-based perception involves wielding an object to discern (non-visually) one or more of its properties. More than 20 years of research using this paradigm suggests, first, that the nonvisual perceptions of a wielded object's spatial and other properties are based on the moments of the object's mass distribution. The moments are invariant over the variations in the forces brought to bear on the objects and on the body's tissues during wielding (Figure 1). Second, special design and analysis procedures have demonstrated that a wide variety of object properties of a wielded object are not only perceivable but are perceived independently of one another ${ }^{13-15}$. Third, perception of distinct properties of an object is constrained by distinct parameterizations of the moments. An elaboration of these general findings permits a framework for considering dynamic touch in clinical populations.

The properties perceived by dynamic touch include the whole length of a rod, partial lengths fore and aft of the hand, center of percussion, orientation (of the branch of an L) relative to the hand, position of the grasping hand relative to the rod, and heaviness of the rod. Instructed to perceive property $\mathrm{P}_{\mathrm{i}}$ the participant seemingly assembles the haptic system into a specific subsystem $h_{i}$ such that $P_{i}=h_{i}$ (moments). Collectively, mechanoreceptors, the network of soft tissues in which they are embedded, and the attendant neural nets seemingly implement (softly assemble) distinct functions $h_{i}$ of the moments that deliver distinct intended perceptions of $\mathrm{P}_{\mathrm{i}}^{16,17}$.

For most of the research to date on dynamic touch, the second moment in the form of the inertia tensor has been the primary mechanical variable of interest. It does not necessarily act alone, however, in constraining the perception of the properties of grasped objects and the perception of limb orientation. The zero and first moments can also enter into $h_{i}$ either in conjunction with the inertia tensor or separately depending 
upon experimental circumstances ${ }^{18-20}$. For compactness, $\mathrm{P}_{\mathrm{i}}=\mathrm{h}_{\mathrm{i}}$ (moments) in the present article will be examined through the experimental data that have identified $h_{i}$ in terms of the inertia tensor. For example, whereas whole length perception can be expressed as a power function of eigenvalues of the inertia tensor ${ }^{21}$, and perception of how the object is oriented in the hand is a function of an eigenvector ${ }^{22}$, perception of the partial length in front of the hand can be expressed as a function of an eigenvalue and an eigenvector ${ }^{23,24}$.

The understanding of dynamic touch summarized above applies not only to how one perceives objects and implements, but also to the traditional concern of how one perceives the spatial orientation of the body and its limbs. The mass distribution of the body, its limbs, and its limb segments are describable through inertia tensors, defined about the respective rotation points in the joints. A number of studies have suggested that perception of limb orientation may be constrained by the directions of the eigenvectors ${ }^{25-27}$. For example, the mass distribution of participants' arms can be manipulated by attaching splints with weights positioned to reorient eigenvectors of the limbplus-splint up, down or not at all. Participants asked to match the position of the two forearms align the eigenvectors and not the geometrical axis of the arms ${ }^{27}$. Such a finding supports the view that the position sense is based on inertial eigenvectors (or the first moment vector; see van de Langenberg, Kingma and Beek ${ }^{20}$ ) and not on joint angles as is traditionally supposed.

The aforementioned experimental findings indicate that mechanical quantities that do not vary with the act of wielding an object or moving a limb constitute the informational basis for muscle-based perception. How does the haptic subsystem of dynamic touch detect such mechanical quantities? During wielding, the torques and motions of the limb-plus-object system generate a structured array of deformation of the muscles and connective tissues of the body. We suppose, first, that invariants of deformation patterns stand in lawful correspondence to the previously described mechanical invariants of the limb-plus-object dynamics (Solomon and Turvey ${ }^{28}$ ) and, second, that the invariants of tissue deformation are extracted by mechanoreceptors embedded in muscles and connective tissue yielding perception of the intended object properties.

\section{General principles underlie perception by dynamic touch $: \because 8$.}

The simple experimental methodology typically used to investigate muscle-based perception allows the expression of a general principle: muscle-based perception relies on physical invariants that are extracted from the time-varying deformation patterns in the musculoskeletal system. An implication of this principle is that in order to perceive a certain object property (e.g., length), perceivers must simply establish a dynamic relation to the object that reveals the invariant mass moments (e.g., $I_{1}$ ) specifying that property. The details of the interaction do not matter as long as it leaves invariant the relevant mechanical quantities. Two important predictions follow from this assertion: first, muscle-based perception of object properties should not be dependent on the specific parts of the body or on the specific movement patterns used to interact with the object; second, muscle-based perception should not rely on sensations arising from local skin contact with the object.

Evidence supporting prediction (first) comes from several sources. Perceiving object length is unaffected by variation in the neuromuscular patterning associated with an overhand or underhand grip ${ }^{12}$, a pinch grip or full hand grasp ${ }^{29}$. Participants asked to judge the length of hand-held objects by wielding them about the wrist, elbow or shoulder are constrained by the largest principal moment of inertia defined at the wrist, regardless of the joint used for wielding ${ }^{30}$. Even more dramatically, perception of object length is equivalent whether that object is moved about the wrist joint or the ankle joint despite marked neural, anatomical and experiential differences between upper and lower extremities ${ }^{31,32}$. In all these cases, the perceptual outcome is related to the objects' mass moments. These findings suggest that muscle-based perception requires that the perceiver-plus-object dynamics reveal the invariant mechanical quantities specific to the to-be-perceived property. In support of prediction ( first), the perceptual outcome does not seem to be a function of which part of the anatomy is used or which space-time trajectories are used to generate such dynamics.

Evidence for prediction (second) comes from studies showing that individuals are able to perceive the properties of objects that are not directly in contact with their body ${ }^{33}$. For example, probing and wielding a rod with one hand, two hands, hand+knee, hand+axle or with a stick+axle result in the same perceptual outcome, namely, perceived length is a single-valued function of mass moments ${ }^{33,34}$. There is no neural substrate in the stick, of course, hence, no sensations arising from local contact with the to-be-perceived rod. As suggested by prediction (second), therefore, muscle-based perception does not seem to depend on local sensations. This finding indicates that what qualifies as a receptive surface is not necessarily the neural substrate but simply susceptibility to the kinds of interactions needed to reveal the invariant mass moments relevant to the perceptual task. The types of interactions that a stick undergoes while contacting and wielding another rod are comparable to those of a hand, knee or foot undergoing the same dynamics. The tissue deformation consequences of such dynamics do not need to be extracted locally, that is, at 
the point of contact. Muscle-based perception seems to take advantage of the global deformation field of the body. How might the haptic substrate be characterized so as to allow the non-local perceptual achievements of dynamic touch implied by predictions one and two?

\section{The architecture of the haptic substrate $\because: \therefore$.}

The classical characterization of haptic perception focuses on signals transmitted linearly from mechanoreceptors to spinal neurons to somatosensory areas in the brain. These signals refer to stresses and strains of individual muscles, ligaments, joint capsules and fascia. However, deformations of individual tissues bear no reliable relation to those properties that the haptic system perceives reliably (i.e., macroscopic properties of objects and limbs relevant to the control of action). For example, different deformation patterns of an individual muscle will result from wielding a given object in different ways. Analogously, the same deformation pattern may result from interaction with different objects. Hence, if mechanoreceptors only inform about the state of individual structures, the nervous system is left with the intractable job of registering individual inputs, combining them, and inferring their most probable cause (e.g., the most likely object that could lead to a particular pattern of mechanoreceptor input). Such explanations of the achievements of dynamic touch necessarily ascribe to the nervous system knowledge about the world and the body without heed to how such knowledge is obtained (Pagano and Turvey ${ }^{35}$ ).

It has been argued, however, that individual anatomical structures do not represent the proper architectural unit for understanding the organization of mechanoreptors and, hence, do not constitute the best characterization of the haptic substrate. An alternative, functional architectural unit for understanding the distribution of the mechanoreceptors points to connective tissue (more specifically, the fascia) and muscle tissue organized in series ${ }^{36}$. The functional architectural unit for mechanoreceptor distribution suggests a necessarily more cooperative and molar take on the organization of the haptic substrate.

A number of anatomical studies provide evidence that the musculoskeletal system is constituted by a nesting of interconnected tissues that could support the cooperative and molar organization of the haptic substrate implicated in the preceding. For example, dissection studies of Van Mameren and Drukker (cited by Van Der Wal ${ }^{36}$ ) have shown connectivity of soft tissues (muscular and passive) in the lateral cubital region. Perhaps surprisingly, it appears that only a few colagenous and muscle fibers run from bone to bone; more generally, the apparatus is complex, consisting of layers of muscular and other soft tissues (e.g., ligaments and capsules), interconnected by fascia tissue. Moreover, such coherence is seen not only within but also across different regions of the body ${ }^{37,38}$. These findings suggest that the haptic substrate should be seen as an integrated whole, in which a local mechanical event (e.g., wielding of a hand-held object) generates an array of tissue deformation at the level of the entire musculoskeletal system specific to that event.

In many respects, such a characterization of the haptic substrate is consistent with what has been referred to as the biotensegrity model of the musculoskeletal system ${ }^{39}$. Tensegrity structures are unique mechanical systems that derive their support function and mechanical stability from continuous tension and local intermittent compression. Considering the musculoskeletal system, the bones can be thought of as the compression members, which are embedded in a network of tightly interconnected soft tissue (muscles, ligaments, and fascia) that constitute the tension-bearing structures.

A tensegrity structure is constantly in balance with its external and internal force environments. Such force balance is guaranteed by a baseline level of tension, or pre-stress, established over the tension-bearing elements. Pre-stress removes any slack in the system and makes it immediately responsive to mechanical stress; consequently, a force applied locally can potentially be sensed globally ${ }^{40}$. As a result of pre-stress, local activities occurring at multiple parts of the structure naturally coordinate to re-establish force balance should it be disturbed by mechanical forces within and adjacent to the system. Thanks to this force-balance constraint, the global deformations of a tensegrity structure are lawfully related to such mechanical forces and, hence, informative about them. Of relevance for present purposes, recall that muscle-based perception of object properties results from the detection, by the haptic system, of invariants of global deformations generated by active interactions with objects. Although multiple mechanoreceptors are active at multiple locations during such interactions, rapid integration to a few degrees of freedom would be possible with the force-balance property allowed by a biotensegrity architectural organization ${ }^{41,42}$. In support of this conjecture, the forcebalance property of tensegrity structures has been shown to be the basis for mechanosensation in the cell ${ }^{43,44}$.

The hypothesized architectural organization of the musculoskeletal system suggests, therefore, that the basis of haptic perception of object properties is not activity at the level of individual receptors or at the point of contact with the object. Instead, it is the pattern of mechanoreceptor activity at the level of the whole system that constitutes the substrate for haptic perception, the level at which the force balance is established. The conjectured registration of deformation patterns at a scale encompassing the proprioceptive substrate as a whole 
is consistent with the non-local perceptual achievements of dynamic touch, namely, successful perception of object properties with non-neural implements and stability of perceptual outcomes over variations in the style of interaction with the objects and in the effectors used in such interactions.

\section{Sensory and motor challenges to perception by dynamic touch $: \because$.}

A number of conditions compromise the body's ability to register stimulation or to execute movements so as to obtain stimulation. These can be gradual, as with the normal consequences of aging, or sudden, as with the aftermath of a spinal cord injury. In either case, the substrate has been altered. What becomes of its ability to extract invariants from the tissue deformation that accompanies interacting with objects?

If the non-local perceptual achievements of dynamic touch, consistent with the tensegrity characterization of the haptic substrate, generalize to individuals with sensory and/or motor impairments, deficits in muscle-based perception in these populations might be far from obvious. For example, individuals presenting motor deficits might interact with objects in alternative ways (i.e., using different parts of the body or different kinematics) and still be able to detect the relevant information for muscle-based perception of objects' properties. Perhaps more dramatically, individuals who cannot feel an object where it contacts their skin might still be able to wield it and
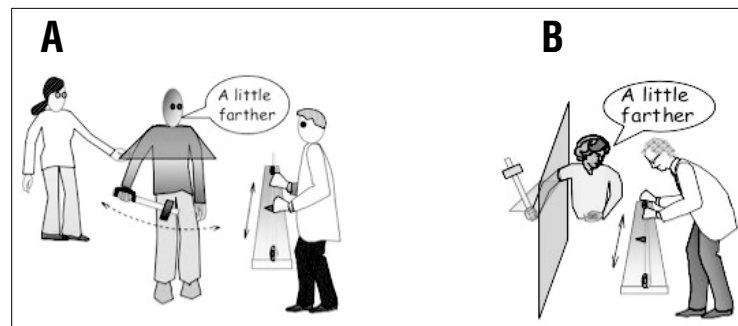

C

D
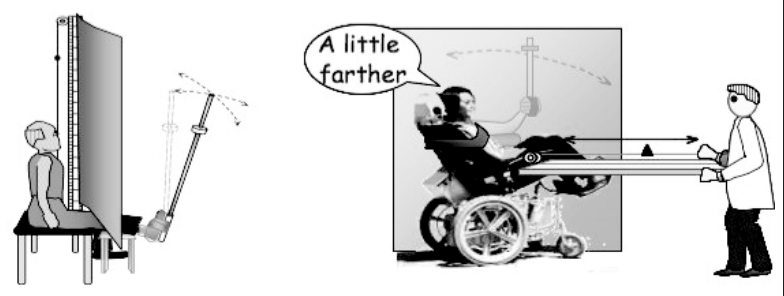

Figure 3. Experimental set-up for the participants of the four case studies: (A) motor challenge to dynamic touch: atypical kinematics due to stroke; (B) sensory challenge to dynamic touch: an insensate arm; (C) sensory challenge to dynamic touch: insensate feet; (d) sensory and motor challenges to dynamic touch: insensate limb and loss of motor control due to spinal cord injury. successfully perceive its inertia-based geometric properties to the extent that the global deformation field is structured lawfully by their movements. In other words, muscle-based perception is expected to be more resistant to sensory and motor challenges than other forms of haptic perception. We turn to a summary of research on dynamic touch by individuals with compromised sensory and motor systems that gives empirical support to this hypothesis.

\section{The general methodology for dynamic touch $: \therefore$.}

Muscle-based perception has been evaluated in individuals with different sensory and/or motor deficits, both implied (e.g., age-related) and diagnosed (e.g., neuropathies, stroke, spinal cord injury). In the general methodology, the task of participants is to wield objects (e.g., wooden rods, rods with attached masses, or common objects such as tennis rackets) hidden from view and indicate their length by magnitude production (Figure 3). If the participant is able, he or she manipulates a pulley to adjust the position of a visible marker to indicate perceived extent. Alternatively, the participant may instruct one of the experimenters to adjust the marker. Objects differ in length and, typically, mass distribution. The latter can be manipulated by gripping the object at different locations along its length or by attaching metal disks at different distances from the proximal end. Such manipulations produce sets of rods that have equal length and mass but different mass moments.

The success of perception (e.g., by impaired and unimpaired limbs) can be compared in several ways. Average deviation $(\mathrm{AD} \%)$ provides a measure of reliability of responses expressed as a percentage of mean $\mathrm{L}_{\mathrm{p}}$. Averaged across all rods, $\mathrm{AD} \%$ yields a measure of consistency for a limb. Mean root square error (MRS\%) provides a parallel measure of accuracy, this time expressed relative to actual length. (MRS is the standard RMS configured in the form of a Weber fraction to facilitate a comparison of values across different settings. See Hajnal et al. $\left.{ }^{32}\right)$.

Higher values of $\mathrm{AD} \%$ and MRS\% indicate lower levels of reliability and accuracy, respectively.

An additional comparison index is made possible by the manipulation of the mass distribution. As noted, in typical experiments using such objects, perceived length is related to the second moment of mass distribution by a power function. The particular form of the power function is $\mathrm{Lp} \propto \mathrm{I}_{1}{ }^{1 / 3}{ }^{21}$. Therefore, both the fact of an inertial dependency and the form of that dependency can be evaluated for participants presenting sensory-motor deficits. In summary, the employed methodology 
allows a rich evaluation of participants' muscle-based ability to perceive linear extent.

\section{Aging and dynamic touch $: \therefore$.}

Declines in visual and auditory systems with aging are wellrecognized consequences of aging. A parallel decline in touch should not be surprising. Physically, it is the case that the morphology of mechanoreceptors changes and their distribution density decrease ${ }^{45,46}$. Average muscle mass and strength decline (e.g., Aniansson et al. ${ }^{47}$ ). And there are, indeed, sensory consequences: spatial acuity (e.g., Thornbury e Mistretta ${ }^{48}$ ) and vibratory sensitivity (e.g., Kenshalo ${ }^{49}$ and Schaumberg, Spencer and $\mathrm{Ochoa}^{50}$ ) decline with age. But are such changes manifested in perception by dynamic touch?

Community-dwelling, healthy active adults ranging from 62 to 89 years old (with an average age of 70 ) were recruited from a pool of people who regularly participated in the social activities of a Senior Center. Eight individuals grasped occluded rods (length $\mathrm{L}=60,90$, or $120 \mathrm{~cm}$ ) at positions intermediate (1/4L, $1 / 2 \mathrm{~L}$, or $3 / 4 \mathrm{~L}$ ) between the ends of the rods. The question posed on a trial was either "How far could you reach with the rod portion in front of the hand?" or "How far could you reach with the whole rod if you were to hold it at its end?"51. The predicted pattern is rationalized by inertial considerations: Whereas $\mathrm{L}_{\text {PARTIAL }}$ is effectively constrained by moment of inertia in front of the hand, $\mathrm{L}_{\mathrm{WHOLE}}$ is constrained by moment of inertia of the whole rod. Consequently, $\mathrm{L}_{\text {PARTIAL }}$ should increase from $1 / 4$ to $1 / 2$ to $3 / 4$ (i.e., as the grip gets farther from the front), but $\mathrm{L}_{\mathrm{WHOLE}}$ should be equal for $1 / 4$ and $3 / 4$ grips both of which should be larger than the $1 / 2$ grip. The predicted pattern is typically found for young adults (e.g., Carello, Santana and Burton ${ }^{24}$ ) and was replicated with older adults. Moreover, the distinct tensorial dependencies for perceived whole length and perceived partial length was replicated as well. Namely, whereas perceived partial length depends on both $\mathrm{I}_{\mathrm{xx}}$ and $\mathrm{I}_{\mathrm{yz}}$, perceived whole length depends only on $\mathrm{I}_{\mathrm{Xx}}$. Participants from the same demographic evaluated different objects in terms of the questions "How long is the object?" or "Where on the object would you like to hit a ball?" Whether the objects were tennis rackets (ranging in length from 53 to $73 \mathrm{~cm}$ ) or wooden rods $(44,60$, and $75 \mathrm{~cm})$ with attached metal rings (at $1 / 3$ or $2 / 3 \mathrm{~L}$ ), perceived sweet spot (center of percussion) was distinguished from perceived length. Both rods and rackets showed the familiar 1/3 scaling of perceived length to $\mathrm{I}_{\mathrm{Xx}}$ albeit with a lower intercept than was found for young participants ${ }^{52}$. Reliabilities of $7 \%$ and MRS of $23 \%$ are comparable to those found under the same circumstances for young adults ${ }^{53}$. The foregoing results suggest that perception of object properties by dynamic touch is equivalent in young and older adults despite the differences in the number and morphology of mechanoreceptors supporting the control of exploratory movements.

\section{A motor challenge to muscle-based perception: case study of atypical kinematics due to stroke $: \because$.}

The LW, a 64-year old male who was three years post Cerebral Vascular Accident (stroke), presented minimal active movements of the wrist. He was able to move his arm using the shoulder joint, albeit with significant restrictions of flexion, abduction and external rotation. He had no neuropathic impairments (e.g., two-point discrimination, detection and location of light touch were intact). Hence, even though LW could feel objects that were placed in his hand, he did not have at his disposal all the mechanical degrees of freedom that are typically available to healthy individuals to move those objects.

Due to significant movement restriction, LW was able neither to grasp the rods tightly in his affected hand nor to wield about the wrist (as in the standard methodology). Instead, a rod was secured to his hand with an elastic band and wielding was performed primarily through shoulder movements. Experimenters requested that LW use a similar wielding style with the unimpaired limb, although he was able to grasp the rod firmly in that case. A horizontal occlusion screen held by one of the experimenters blocked his view of the rods (3A).

The mean perceived lengths with the affected and unaffected hands were 36,53 , and $73 \mathrm{~cm}$ and 35,49 , and $63 \mathrm{~cm}$, respectively, for the 45,60 , and $75 \mathrm{~cm}$ rods. Values of $\mathrm{AD} \%$ (approximately $8 \%$ ) and MRS\% (approximately 20\%) did not differ between affected and unaffected $\operatorname{limb}^{54}$ and compare favorably to those obtained with young adults without movement disorders ${ }^{55}$. These findings indicate that LW's muscle-based capability to perceive the lengths of objects was generally preserved, regardless of the significant movement restrictions of the upper limb. The successful perception of object length by healthy adults using a variety of movement styles provides a context for this result.

For both LW's impaired and unimpaired limbs, a power function related perceived length $\left(\mathrm{L}_{\mathrm{p}}\right)$ to the maximal principle moment of inertia $\left(I_{1}\right): L_{P}=a\left(I_{1}\right)^{b}$. The slopes of the functions were similar for the affected and unaffected limbs. Together with the significant difference between $\mathrm{AD} \%$ and MRS\%, these findings suggest that, for LW too, perception is not based on metrical length but on a higher-order invariant of the wielding dynamics specific to length. In addition, it suggests that the principles underlying muscle-based perception in the presence 
of motor impairment are the same as the ones previously identified in individuals without motor dysfunction.

\section{A sensory challenge to dynamic touch: case study of an insensate arm $: \because$.}

The participant was AA, a 40-year old female, presenting peripheral neuropathy secondary to lesions to the lower cervical and upper thoracic segments on the left of the dorsal column system. At the time of the experiment, AA experienced insensitivity in the left arm, extending from the hand to the elbow with some involvement of the shoulder. As a result, she could not identify an object contacting her hand, nor tell whether an object was in the hand even when manipulating it. (A stereognosis test with 3-dimensional numbers yielded a score of zero with the affected limb and a score of $100 \%$ with the unaffected $\mathrm{limb}^{55}$ ). These two inabilities are strong indicators of compromised cutaneous and haptic touch within the dorsal column system. Despite her sensory deficits, she was able to grasp the rods firmly when requested and wield them from the wrist, following the typical methodology. However, because she was unable to manipulate the pulley with her affected arm, one of the experimenters did so at her behest (Figure 3B). Performance with both limbs was evaluated.

For the 45, 60 and $80 \mathrm{~cm}$ rods, the right (unaffected) hand yielded mean $\mathrm{L}_{\mathrm{p}}$ of 48,56 and $79 \mathrm{~cm}$; the left (affected) hand yielded mean $\mathrm{L}_{\mathrm{p}}$ of 35,48 and $60 \mathrm{~cm}$. Reliability and accuracy of AA's reports were lower for the impaired limb. The observed difference does not seem to be a function of sensory deficits in the affected limb but, rather, a result of an unusually high performance of the unaffected limb ${ }^{55}$. The values of mean AD\% and MRS\% for the unimpaired limb are considerably smaller than those obtained for most unimpaired participants. AD\% and MRS\% of the insensate limb compares favorably to those obtained with young adults without movement disorders. Hence, even though AA could not feel the objects in her hand, she was able to get accurate and reliable impressions of the length of the objects by wielding.

For both AA's insensate and intact limbs, length judgments varied systematically with changes in the rotational inertia of the objects about the wrist. The slopes in double logarithmic coordinates were the same (0.35) for the two hands, and in close agreement with the value expected from dimensional analysis and standard experimental observation. These findings indicate that the informational support for the task is the same and relatively indifferent to complete loss of cutaneous and haptic touch of the hand in direct contact with the rod. This result suggests that wielding deforms the tissues of the body, not just those around the hand, due to the interconnected nature of the musculoskeletal system ${ }^{36}$. In other words, wielding induces a deformation field invariantly related to the properties of the rod, which may be exploited by the preserved field-like structure of mechanoreceptors. Interestingly, the intercept for the two hands differed, with that for the insensate limb being lower than for the unaffected limb. This echoes the direction of difference between older and younger participants for perceived length and sweet spot ${ }^{52}$ where it might be assumed that older participants have begun to lose cutaneous sensitivity in the extremities.

\section{A sensory challenge to dynamic touch: case study of insensate feet $: \therefore$.}

The participant was a 72-year old male, WP, presenting peripheral neuropathy secondary to diabetes. At the time of the experiment, he presented complete insensitivity from foot to midcalf, bilaterally: cutaneous touch and haptic touch were profoundly affected. In order to evaluate the muscle-based capability of WP's dominant lower limb, a handle was attached firmly to the foot (by sheathing the individual's foot and shoe in plastic and taping the handles flush with the heel). The rods were slipped into the handle and the participant was asked to wield them using his ankle (Figure 3C). Estimates of rod length were provided by moving a marker on the report apparatus. W could not feel when a rod had been attached to his foot, so experimenters would tell him, on every trial, when to start wielding. Previous studies have shown that the muscle-based perceptual capabilities of hand and foot are comparable in unimpaired individuals. Hence, dynamic touch capability of W's intact dominant upper limb was also assessed for comparison.

For the 60, 80 and $100 \mathrm{~cm}$ rods, the right hand yielded mean $\mathrm{L}_{\mathrm{P}}$ of 66,74 and $78 \mathrm{~cm}$; the right foot yielded mean $\mathrm{L}_{\mathrm{P}}$ of 52,65 and $78 \mathrm{~cm}$. The values of $\mathrm{AD} \%$ were similar for hand and foot (6.57 and $7.15 \%$, respectively). These values are similar to those demonstrated by unimpaired individuals ${ }^{31}$. MRS\% was smaller for the hand (10.49\%) when compared to foot (19.72\%). Such a difference is most likely not related to the neuropathy given that the values of MRS\% obtained for the foot were lower than those previously observed for unimpaired individuals performing the same task ${ }^{31}$. Clearly, $\mathrm{W}$ was able to perceive the length of objects by wielding with the foot, despite his sensory deficits.

We observed systematic variations of the length judgments with changes in the rotational inertia of the objects about the wrist and ankle. The slopes in log-log plots are different for the hand and foot, but both are in the range commonly observed in previous research ( 0.33 and 0.22 for foot and hand, respectively). 
These findings indicate that loss of sensitivity to touch on the foot and lower leg does not prevent sensitivity to the inertial properties of objects. Once again, however, the intercept of the regression is lower for the insensate limb.

\section{Sensory and motor challenges to dynamic touch: insensate limb and loss of motor control due to spinal cord injury $: \because 8$.}

The muscle-based perceptual capability of individuals with combined sensory and motor deficits has also been investigated $^{56}$. Six individuals with cervical spinal cord injury (ranging from $\mathrm{C} 4$ to $\mathrm{C6}$ ) were diagnosed with quadriplegia. They presented loss of cutaneous sensitivity and significant impairment in motor coordination such that they could not feel the objects in their hand and had only very limited motor capabilities to explore those objects' properties (primarily by thrashing movements; Koike 2007, personal communication, July). Due to the significant movement restriction, participants were not able to hold and wield the rods against gravity. Instead, a rod was secured to each individual's right hand with an elastic band and a curtain was used to hide the object from view (Figure 3D). Two different wielding styles were used: two-joint (elbow and wrist) or three-joint (shoulder, elbow and wrist) rotations. The movement styles were differentiated by the degree to which the limb was strapped to the individual's wheelchair.

The mean $\mathrm{L}_{\mathrm{p}}$ for the 50,65 and $90 \mathrm{~cm}$ rods were 65,78 , and $97 \mathrm{~cm}$. Even though the authors did not report reliability and accuracy measures, the judgments were in the ballpark of actual length as commonly observed in previous studies with unimpaired participants. These findings indicate that the musclebased capability to perceive the length of objects was generally preserved in the six participants, regardless of the pronounced sensory and motor impairments of the upper limb. A systematic variation of the length judgments with changes in the rotational inertia of the objects was also observed for both styles of wielding. The slopes in log-log plots were similar for the twojoint (0.28) and three-joint condition (0.21) for the two hands, indicating that the kinematics of wielding did not affect $L_{P}$ or its informational basis. These findings indicate that the sensory and motor impairments presented by the participants did not prevent attunement to the invariants of the wielding dynamics, specific to the to-be-perceived property. The present case study lends further support to the idea that muscle-based perception is not a local process - the participants' very restricted field of preserved mechanoreceptors and attendant neural and fascia nets were sufficient to support its basic functioning.
The foregoing research indicates that the fundamental muscle-based perceptual capabilities of insensate limbs are generally preserved. It has been argued that perception via neuropathic limbs may be considered analogous to perception via non-neural attachments including hand-held implements and prosthetic devices ${ }^{57}$. The results just presented are consistent with such an argument. The mechanism supporting perception with intact, insensate limbs or non-neural implements seems to be one and the same: detection of movementproduced mechanical invariants.

The clinical samples and case studies suggest that musclebased perception is robust enough to overcome not only motor but also substantial sensory challenges. The findings from these studies indicate that muscle-based perception might be the most fundamental form of haptic perception. Such an assertion is not surprising in light of the basic functional capabilities it underlies and the devastating consequences of its loss.

\section{Implications for rehabilitation $\because: 8$}

A general conclusion of our summary of a variety of clinical case studies of dynamic touch is that this subsystem of the haptic perceptual system is not markedly impaired by either sensory or motor challenges. While initially surprising, there are enough suggestions in the literature on healthy adults that foretold such robustness. As noted, the perceptual capabilities of foot/ankle are comparable to those of hand/wrist despite pronounced physiological, morphological, and experiential differences between those appendages ${ }^{31,32,55}$. Considering lower extremity relative to upper extremity, mechanoreceptors are fewer, skin is thicker, muscles are larger, and dexterous manipulations are not a typical activity. Nonetheless, accuracy, reliability, and inertial dependencies are the same. The commonalities reported for any two-point stabilization of a $\operatorname{rod}^{34}$ makes a similar point: The specific deformed tissue in not important, the deformation is. Certainly the rarity in the medical literature of complete haptic loss is telling. The dozen or so documented cases - in contrast to the millions of cases of total visual or hearing loss - speaks to the robustness of the haptic substrate. As long as something is preserved, invariants can be extracted.

What possible implications are there, then for rehabilitation? Are we actually considering a system not in need of remediation? Certainly functional capabilities of dynamic touch are generally preserved whether or not individuals are aware of their preservation. Keep in mind that most of our participants were quite surprised at their success. Participants are forced to respond in a situation for which there is no consequence of a wrong answer. But they clearly lack 
the confidence or inclination to control actions with objects that might break or with an awkwardness that would prove embarrassing socially. In addition, the smaller intercept demonstrated by elderly individuals and the ones with sensory deficits indicate that muscle-based sensitivity, even though generally preserved, might be decreased in these populations. Hence, one could imagine rehabilitation efforts directed at heightening both awareness of and sensitivity to the distributed deformation field that allows perception of object and limb properties relevant for the control of action. Below we will suggest possible intervention strategies that, although grounded in the theory of muscle-based perception just reviewed, are speculative and in need of scientific scrutiny.

An ecological approach to rehabilitation of individuals with sensory and motor deficits would focus not only on the organism but also on the environment that supports his or her actions. One avenue for rehabilitation, directed at the organism side of the organism-environment system, is to help individuals use their preserved sensitivity to the inertial properties of objects functionally. Training individuals to explore and use objects with varied mass distributions may increase their awareness of the opportunities that different objects provide for patterning muscular forces in the context of different task requirements. What makes certain objects unwieldy (or unwieldy for particular purposes) seems to be constrained by their inertia tensors ${ }^{58}$. Attuning individuals to movement-based constraints on dynamic touch may allow more coordinated and efficient movement patterns to emerge.

Increased sensitivity of the haptic subsystem of dynamic touch may result from interventions focusing on the mechanical responsiveness of the deformation field to stress. There is evidence that spindle afferent discharges are related to changes in the elastic properties of the tissues in which they are embedded ${ }^{59}$. Specifically, it has been reported that stiffer muscle-tendon elements ensure that the muscle spindles are more sensitive to imposed external forces $^{60}$. Strength training has been shown to increase the stiffness not only of muscular but also of connective tissues surrounding the targeted joint ${ }^{61,62}$. Hence, such intervention (targeting muscles of the whole body, not only of the injured limb) could possibly lead to increased responsiveness of the distributed deformation field and, consequently, to better attunement to the inertial properties of objects and limbs relevant to the control of action. Supporting this conjecture is evidence that greater muscle strength is related to better muscle-based proprioception both in unimpaired populations $^{63}$ and in populations of individuals with ligament injury ${ }^{64}$.
As noted, the interventions suggested in the preceding address the organism side of the organism-environment system. A complementary tack would focus on the environment side. Just as athletes benefit from movement-centered redesign of their implements (e.g., hockey sticks fashioned to reduce I so as to increase stick speed; e.g., Haas and Mollner ${ }^{65}$ ) so, too, can clinical populations benefit from movement-centered redesign of ordinary implements. Namely, so design objects and tools as to maximize tissue deformation relevant to controlling objects. Crudely, bottom heavy objects are not only more stable but also deform tissue in a way that points to upright. One might conjecture that easily orientable objects are easier to control. For example, a glass may be weighted more at the bottom so that its vertical position is more readily revealed, which might prevent the liquid from being spilled when the glass is being carried around.

There is evidence that healthy individuals can perceive the suitableness of an implement for the performance of different functional activities (e.g., hammering versus poking) by detecting its mass distribution by dynamic touch. Hence, in order to facilitate their functional use, objects can be built such that their mass distributions better support the control of the desired action. For instance, a cylindrical rod with mass concentrated close to the hand seems to be adequate for precision tasks, such as poking ${ }^{66}$. On the other hand, an object of the same shape and size, with mass concentrated far from the hand, facilitates transfer of momentum from the hand to the environment, constituting a suitable tool with which to strike objects. In fact, it has been demonstrated that children produce more stable movement trajectories when striking a target with objects having this sort of mass distribution (Fitzpatrick P 2008, oral communication, February). Hence, it is possible that the movement trajectories of individuals with sensory and motor deficits might be improved if the mass distributions of objects specify more distinctly the patterns of forces necessary to move them in the desired way.

In summary, an implication of the research on dynamic touch summarized in this paper, along with the theoretical approach that motivated the research, is that rehabilitation of individuals with sensory deficits should go beyond the locally impaired sensory apparatus. The fact that an insensate limb can be used to explore and perceive the properties of objects it contacts indicates that muscle-based perception exploits the body structure of mechanoreceptor support and does not rely solely on the stimulation arising from local movement and tissue contact. These strategies involve not only addressing and optimizing global capabilities of the haptic system but also manipulating properties of the environments (e.g., tools and prosthetic devices) to which the globally preserved 
mechanoreceptor support is sensitive. Future research efforts may seek to evaluate whether these types of interventions can, in fact, promote improvements in muscle-based perception and coordination of action.

\section{References}

1. Gibson JJ. The senses considered as perceptual systems. Boston: Houghton Mifflin; 1966.

2. Turvey MT. Dynamic touch. Am Psychol. 1996;51(11):1134-52.

3. Turvey MT. Dynamics of effortful touch and interlimb coordination. J Biomech. 1998;31(10):873-82.

4. Dellon AL. Evaluation of sensibility and re-education of sensation in the hand. Baltimore, MD: Williams and Wilkins; 1981.

5. Dellon AL, Kallman C. Evaluation of functional sensation in the hand. J Hand Surg [Am]. 1983,8(6):865-70.

6. Moberg E. Objective methods for determining the functional value of sensibility in the hand. J Bone Joint Surg. 1958,40-B(3):454-76.

7. Brink EE, Mackel R. Sensorimotor performance of the hand during peripheral nerve regeneration. J Neurol Sci. 1987;77(2-3):249-66.

8. Sundholm LK, Eliasson AC, Forssberg H. Obstetric brachial plexus injuries: assessment protocol and functional outcome at age 5 years. Dev Med Child Neurol. 1998;40(1):4-11.

9. Yozbatiran N, Der-Yeghiaian L, Cramer SC. A standardized approach to performing the action research arm test. Neurorehabil Neural Repair. 2008;22(1):78-90.

10. Wolf S, Catlin PA, Ellis M, Archer AL, Morgan B, Piacentino A. Assessing Wolf motor function test as outcome measure for research in patients after stroke. Stroke. 2001;32(7):1635-9.

11. Carello C, Turvey MT. Physics and psychology of the muscle sense. Curr Dir Psychol Sci. 2004;13:25-8.

12. Solomon HY, Turvey MT, Burton G. Perceiving extents rod extents by wielding: haptic diagonalization and decomposition of the inertia tensor. $J$ Exp Psychol Hum Percept Perform. 1989;15(1):58-68.

13. Amazeen EL. Perceptual independence of size and weight by dynamic touch. J Exp Psychol Hum Percept Perform. 1999;25(1):102-19.

14. Cooper MM, Carello C, Turvey MT. Further evidence of perceptual independence (specificity) in dynamic touch. Ecol Psychol. 1999;11(4): 269-81.

15. Cooper MM, Carello C, Turvey MT. Perceptual independence of whole length, partial length, and hand position in wielding a rod. J Exp Psychol Hum Percept Perform. 2000;26(1):74-85.

16. Turvey MT. Simplicity from complexity: Archetypal action regimes and smart perceptual instruments as execution-driven phenomena. In: Kelso JAS, Mandel AJ, Shlesinger MF, editors. Dynamic patterns in complex systems. Singapore: World Scientific; 1988.

\section{Acknowledgments $: \because$.}

Preparation of this manuscript was supported by a grant from the Provost's Office at the University of Connecticut.

17. Turvey MT. Action and perception at the level of synergies. Hum Mov Sci. 2007;26(4):657-97.

18. Kingma I, van de Langenberg R, Beek PJ. Which mechanical invariants are associated with the perception of length and heaviness of a nonvisible handheld rod? Testing the inertia tensor hypothesis. J Exp Psychol Hum Percept Perform. 2004;30(2):346-54.

19. van de Langenberg R, Kingma I, Beek PJ. Mechanical invariants are implicated in dynamic touch as a function of their salience in the stimulus flow. J Exp Psychol Hum Percept Perform. 2006;32(5):1093-106.

20. van de Langenberg R, Kingma I, Beek PJ. The perception of limb orientation depends on the center of mass. J Exp Psychol Hum Percept Perform. 2008;34(3):624-39.

21. Fitzpatrick $P$, Carello $C$, Turvey MT. Eigenvalues of the inertia tensor and exteroception by the "muscular sense". Neuroscience. 1994;60(2):551-68.

22. Turvey MT, Burton G, Pagano CC, Solomon HY, Runeson S. Role of the inertia tensor in perceiving object orientation by dynamic touch. J Exp Psychol Hum Percept Perform. 1992;18(3):714-27.

23. Turvey MT, Carello C, Fitzpatrick P, Pagano CC, Kadar E. Spinors and selective dynamic touch. J Exp Psychol Hum Percept Perform. 1996:22:1113-26.

24. Carello C, Santana MV, Burton G. Selective perception by dynamic touch. Percept Psychophys. 1996;58(8):1177-90.

25. Garrett S, Pagano C, Austin G, Turvey MT. Spatial and physical frames of reference in positioning a limb. Percept Psychophys. 1998;60(7):1206-15.

26. Pagano CC, Turvey MT. The inertia tensor as a basis for the perception of limb orientation. J Exp Psychol Hum Percept Perform. 1995;21(5): 1070-87.

27. Pagano C, Garrett S, Turvey MT. Is limb proprioception a function of the limbs' inertial eigenvectors? Ecol Psychol. 1996;8:43-69.

28. Solomon HY, Turvey MT. Haptically perceiving the distances reachable with hand-held objects. J Exp Psychol Hum Percept Perform. 1988;14(3): 404-27.

29. Pagano CC, Kinsella-Shaw JM, Cassidy PE, Turvey MT. Role of the inertia tensor in haptically perceiving where an object is grasped. J Exp Psychol Hum Percept Perform. 1994;20(2):276-85.

30. Pagano CC, Fitzpatrick P, Turvey MT. Tensorial basis to the constancy of perceived object extent over variations of dynamic touch. Percept Psychophys. 1993;54(1):43-54.

31. Hajnal A, Fonseca S, Harrison S, Kinsella-Shaw J, Carello C. Comparison of dynamic (effortful) touch by hand and foot. J Mot Behav. 2007;39(2):82-8. 
32. Hajnal A, Fonseca S, Kinsella-Shaw JM, Silva P, Carello C, Turvey MT. Haptic selective attention by foot and by hand. Neurosci Lett. 2007;419(1):5-9.

33. Peck A, Jeffers RG, Carello C, Turvey MT. Haptically perceiving the length of one rod by means of another. Ecol Psychol. 1996;8:237-58.

34. Carello C, Fitzpatrick P, Domaniewicz I, Chan TC, Turvey MT. Effortful touch with minimal movement. J Exp Psychol Hum Percept Perform. 1992:18:290-302.

35. Pagano $\mathrm{C}$, Turvey MT. Eigenvectors of the inertia tensor and perceiving the orientations of limbs and objects. J Appl Biomech. 1998;14:331-59.

36. Van Der Wal JC. The organization of the substrate of proprioception in the elbow region of rat [Thesis]. Maastricht, The Netherlands: University of Limburg; 1998.

37. Huijing PA. Intra-, extra-, and intermuscular myofascial force transmission of synergists and antagonists: effects of muscle length as well as relative position. Int J Mech Med Biol. 2002;2:1-15.

38. Huijing PA. Muscular force transmission necessitates a multilevel integrative approach to the analysis of function of skeletal muscle. Exerc Sport Sci Rev. 2003;31(4):167-75.

39. Levin SN. The tensegrity-truss as a model for spine mechanics: Biotensegrity. J Mech Med Biol. 2002;2:375-88.

40. Ingber DE. Cellular mechanotransduction: putting all the pieces together again. FASEB J. 2006;20(7):811-27.

41. Fonseca ST, Turvey MT. Biotensegrity perceptual hypothesis: A medium of haptic perception. Paper presented at the North America Meeting of the International Society for Ecological Psychology. Cincinnati, Ohio, June 2224; 2006 .

42. Turvey MT, Fonseca ST. Nature of motor control: Perspectives and issues. In: Sternad D, editor. Progress in motor control: A multidisciplinary perspective. New York: Springer Verlag; 2008. p. 93-123.

43. Ingber DE. Tensegrity I. Cell structure and hierarchical systems biology. J Cell Sci. 2003;116(Pt 7):1157-73.

44. Ingber DE. Tensegrity II. How structural networks influence cellular information processing networks. J Cell Sci. 2003;116(pt 8):1397-408.

45. Bolton CF, Winkelmann RK, Dyck PJ. A quantitative study of Meissner's corpuscles in man. Neurol. 1966;16(1):1-9.

46. Cauna N. The effects of aging on the receptor organs of the human dermis. In: Montagna W, editor. Advances in biology of skin: Aging (vol. 6). New York: Pergamon; 1965.

47. Aniansson A, Sperling L, Rundgren A, Lehnberg E. Muscle function in 75year-old men and women. A longitudinal study. Scand J Rehab Med Suppl. 1983:9:92-102.

48. Thornbury JM, Mistretta CM. Tactile sensitivity as a function of age. J Gerontol. 1981;36(1):34-9.

49. Kenshalo DR Sr. Somesthetic sensitivity in young and elderly humans. J Gerontol. 1986;41(6):732-42.
50. Schaumberg HH, Spencer PS, Ochoa J. The aging human peripheral nervous system. In: Katzman R, Terry R, editors. The neurology of aging. Philadelphia: F.A. Davis Co; 1983. p. 111-22.

51. Carello C, Thuot S, Turvey MT. Aging and perception by dynamic touch. In: Grealy M, Thomson I. Studies in perception and action V. Mahwah, NJ: Erlbaum; 1999. p. 5-88.

52. Carello C, Thuot S, Turvey MT. Aging and perception of a racket's sweet spot. Hum Mov Sci. 2000;19:1-20.

53. Carello C, Thuot S, Andersen KL, Turvey MT. Perceiving the sweet spot. Perception. 1999;28(3):307-20.

54. Silva P, Hajnal A, Harrison S, Kinsella-Shaw JM, Bubela D, Carello C. Perceiving object length by dynamic touch after a stroke: a case study. In: Cummins-Sebree S, Riley M, Shockley K, editors. Studies in Perception \& Action IX. Mahwah, NJ: Erlbaum; 2007. p. 147-50.

55. Carello C, Kinsella-Shaw J, Amazeen EL, Turvey MT. Peripheral neuropathy and object length perception by effortful (dynamic) touch: a case study. Neurosci Lett. 2006;405(3):159-63.

56. Koike T, Miyamoto E, Tamagaki T. Dynamic touch in patients with cervical spinal cord injury. In: Cummins-Sebree S, Riley M, Shockley K, editors. Studies in Perception \& Action IX. Mahwah, NJ: Erlbaum; 2007. p. 151-4.

57. Pagano CC. The role of the inertia tensor in kinesthesis. Crit Rev Biomed Eng. 2000;28(1-2):231-6.

58. Carello C, Wagman JB. Mutuality in the perception of affordances and the control of movement. In: Sternad D, editor. Progress in motor control. New York: Springer Verlag; 2008. p. 271-89.

59. Rosant $\mathrm{C}$, Pérot $\mathrm{C}$. An index of spindle efficacy obtained by measuring electroneurographic activity and passive tension in the rat soleus muscle. $J$ Neurosci Meth. 2006;150(2):272-8.

60. Rack PM, Ross HF, Thilmann AF, Walters DK. Reflex responses at the human ankle: the importance of tendon compliance. J Physiol. 1983;344:503-24.

61. Kubo K, Kanehisa H, Fukunaga T. Effects of resistance and stretching training programmes on the viscoelastic properties of human tendon structures in vivo. J Physiol. 2002;538(Pt 1):219-26.

62. Reeves ND, Narici MV, Maganaris CN. Strength training alters the viscoelastic properties of tendons in elderly humans. Muscle Nerve. 2003;28(1):74-81.

63. Goodman M, Marks R. The association between knee proprioception and isotonic quadriceps femoris strength. Physiother Can. 1998;50:53-7.

64. Fonseca ST, Ocarino JM, Silva PL, Guimarães RB, Oliveira MC, Lage CA. Proprioception in individuals with ACL-deficient knee and good Muscular and functional performance. Res Sports Med. 2005;13(1): 47-61.

65. Haas N, Mollner B. Method of forming a one piece hockey stick. U. S. Patent \#6,893,596. 2005.

66. Wagman J, Carello C. Haptically creating affordances: the user-tool interface. J Exp Psychol Appl. 2003;9(3):175-86. 